\title{
Data Reconstruction Coverage Based on Graph Signal Processing for Wireless Sensor Networks
}

This paper was downloaded from TechRxiv (https://www.techrxiv.org).

\section{LICENSE}

CC BY 4.0

SUBMISSION DATE / POSTED DATE

$02-10-2021 / 12-10-2021$

\section{CITATION}

Feng, Jie; Chen, Fangjiong; Cheng, Hongbin (2021): Data Reconstruction Coverage Based on Graph Signal Processing for Wireless Sensor Networks. TechRxiv. Preprint. https://doi.org/10.36227/techrxiv.16725991.v1

DOI

10.36227/techrxiv.16725991.v1 


\title{
Data Reconstruction Coverage Based on Graph Signal Processing for Wireless Sensor Networks
}

\author{
Jie Feng, Fangjiong Chen, Member, IEEE, and Hongbin Chen
}

\begin{abstract}
Sensing coverage is a crucial metric for the quality of service of Wireless Sensor Networks (WSNs). Coverage models have a great impact on sensing coverage of WSNs. However, existing coverage models are simple but inefficient, like the most frequently used disk coverage model, in which a covered point is within the fixed sensing radius of at least one sensor node. Thus, how to develop an efficient coverage model is an essential problem. To this end, in this letter, we propose a novel coverage model without the limitation of the sensor's sensing radius, namely, Data Reconstruction Coverage (DRC). Based on the theory of graph signal processing, the model can jointly reconstruct missing data at unsampled points (which are not covered by any sensors) by using our proposed centralized data reconstruction coverage algorithm which fully exploits the smoothness of temporal difference signals and the graph Laplacian matrix, without increasing the number of sensors. Simulation results based on real-world datasets show that the proposed DRC model has better coverage performance of WSNs compared with the disk coverage model and confident information coverage model typically used in WSNs.
\end{abstract}

Index terms - Wireless sensor networks, data reconstruction coverage, graph signal processing.

\section{INTRODUCTION}

A $\mathrm{S}$ the rapid advancement of Internet of Things (IoTs), Wireless Sensor Networks (WSNs), the basic support for IoTs, have increasingly attracted much attention from researchers. WSNs offer an effective and economical means to perform monitoring tasks over some environments that are dangerous and inaccessible to humans, such as, underwater, rainforest, desert, etc. For WSNs, sensing coverage is one of the most important metrics for the Quality of Service (QoS) [1]. In fact, sensor coverage model is closely related to the performance of sensing coverage. A coverage model with accurate and efficient properties can bring lower network density and energy consumption to WSNs. Next, we will discuss some typical coverage models of WSNs.

Currently, most works use inefficient coverage models to study coverage problems in WSNs, especially Disk Coverage (DC) model [2] in which the sensing field of a sensor node is usually decided by a disk centered at the node with the prescribed sensing radius (usually a few meters or dozens of meters). But, the DC model is inefficient since it only relies on the sensing radius to decide whether to cover or not and does not adequately enable sensors to cooperatively improve the coverage performance of WSNs. Recently, an advanced coverage model, namely, Confident Information Coverage (CIC) model [3], can enable sensors to cover more unsampled points within their correlation range than the DC model, by leveraging the kriging method based on spatial correlation. However, the CIC model is still very similar to the DC one, i.e., adopting fixed radius (correlation range) to decide the coverage range of sensors. When WSNs adopting these models exist sensor malfunctions or unreasonable distribution, missing data at unsampled points (also called coverage holes, which are not covered by any sensors) severely degrade

Manuscript received October 2, 2021. This work was supported by the National Key Research and Development Program of China under Grant 2020YFB1807700 (Corresponding author: Fangjiong Chen).

J. Feng is with the School of Electronic and Information Engineering, South China University of Technology, Guangzhou 510640, China (e-mail: 496294858@qq.com).

F. Chen is with the School of Electronic and Information Engineering, South China University of Technology, Guangzhou 510640, China, and also with the Key Laboratory of Marine Environmental Survey Technology and Application, Ministry of Natural Resources, Guangzhou 510300, China (e-mail: eefjchen@scut.edu.cn).

H. Chen is with the Key Laboratory of Cognitive Radio and Information Processing, Guilin University of Electronic Technology, Guilin 541004, China (e-mail: chbscut@guet.edu.cn). 
the QoS of WSNs. Therefore, in order to improve the coverage performance, developing an efficient coverage model is very necessary. Then, we will introduce an effective technology for data reconstruction at unsampled points, Graph Singal Processing (GSP) [4], to support our proposed coverage model.

In recent years, the theory of GSP has been widely adopted in many practical applications, such as, data reconstruction in WSNs. Since many real-world data tend to be smooth and exist spatio-temporal correlation, the data can be reconstructed by using the theory of GSP. For example, missing data in WSNs (such as, temperature, sea-level pressure, etc.) need to be reconstructed, since these signals (in this letter, the terms "data" and "signal" will be used interchangeably) are easily missed by various reasons, such as, malfunctions or unreasonable distribution of sensors. Therefore, by leveraging the theory of GSP, reconstructing the sensed data at all unsampled points within the acceptable error can enable WSNs to avoid data missing, which is equivalent to expanding the coverage of WSNs without adding any sensors.

In this letter, we propose a novel coverage model based on the theory of GSP, called as, Data Reconstruction Coverage (DRC). According to the definition of our proposed model, the missing data at unsampled points can be jointly reconstructed by our proposed Centralized Data Reconstruction Coverage (CDRC) algorithm, which fully utilizes the smoothness of the temporal difference signals and the graph Laplacian matrix, and unsampled points are data reconstruction covered if the time-average Root-MeanSquare Error (RMSE) is lower than the error threshold required by an application. Unlike currently inefficient coverage models, our proposed model does not rely on the sensing radius or correlation range of sensors, because it only needs the sensed data at sampled points to jointly reconstruct missing data at unsampled points, which is equivalent to improving the coverage of WSNs without increasing the number of sensors. Also, simulation results by using real-world datasets show that the CDR model can significantly improve the coverage performance of WSNs compared with the DC model and CIC model.

\section{Data Reconstruction Coverage}

In this section, based on the theory of GSP, we propose a novel coverage model, named as, DRC. In this model, by applying the theory of GSP, sensed data at unsampled points can be jointly reconstructed. Next, we will give the definition of DRC.

Consider that sensors distributed at sampled points make sensed data, and unsampled points exist missing data due to sensor failures or incomplete distribution. Since sensed data tend to be smooth, the sensed data at unsampled points can be reconstructed within the acceptable error required by an application.

The reconstructed data at an unsampled point $p$ is evaluated by the time-average RMSE which can be computed by

$$
\bar{e}(p)=\frac{\|\hat{\mathbf{x}}-\tilde{\mathbf{x}}\|_{2}}{\sqrt{\mathcal{N}_{d}}} .
$$

where $\hat{\mathbf{x}}=\left(\hat{x}_{1}, \ldots, \hat{x}_{\mathcal{M}}\right)$ is the vector of the reconstructed data, $\tilde{\mathbf{x}}=\left(\tilde{x}_{1}, \ldots, \tilde{x}_{\mathcal{M}}\right)$ is the vector of the true data, $\mathcal{M}$ is the number of time instants, and $\mathcal{N}_{d}$ is the length of the data. Therefore, our objective is to minimize $\bar{e}(p)$, and the solution is shown in Section III-B.

Definition 1: [DRC ( $\Theta$-coverage)] Given a RMSE threshold $\gamma_{t}\left(\gamma_{t} \geq 0\right)$, an unsampled point (target) $p$ (which requires to be sensed) is data reconstruction covered $(\Theta$-covered), if $\bar{e}(p)$ is equal or less than $\gamma_{t}$, i.e., $\bar{e}(p) \leq \gamma_{t}$. Similarly, a sensing field can be said to be $\Theta$-covered, if $\bar{e}(p)$ of each unsampled point $p$ in the field is equal or less than $\gamma_{t}$.

Next, to simply illustrate the difference between the DC model and our proposed model, we introduce an example shown in Fig. 1. Under the DRC model, based on the theory of GSP, the reconstructed data at unsampled point $p_{3}$ can be jointly reconstructed by using the smoothness of the sensed data generated by sensors $\left(s_{1}\right.$ and $\left.s_{2}\right)$ and the graph Laplacian matrix without the limitation of the fixed radius (the sensing radius or correlation range). In contrast, when the DC model or CIC model is used, the sensed data at $p_{3}$ miss because $p_{3}$ is out of the sensing radius $r_{s}$ or correlation range $r_{c}$ of all sensors. Obviously, we can see that the performance of the coverage with the DRC model is better than that of the DC model and CIC model. 


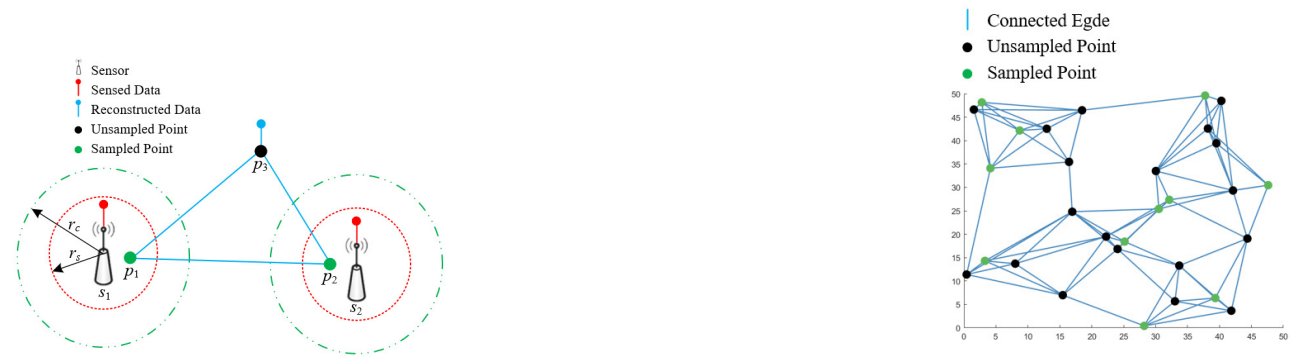

Fig. 1. Coverage comparison between the DRC model and DC model. Fig. 2. Graph constructed by $K$-nearest neighbor algorithm with $K=5$.

\section{Data Reconstruction Coverage Based on Graph Singal Processing}

In this section, we give preliminaries for the graph Laplacian matrix and problem formulation in Subsection III-A; then, in order to minimize the RMSE of each unsampled point, we present a CDRC algorithm by exploiting the smoothness of the temporal difference signals and the graph Laplacian matrix in Subsection III-B; finally, we analyze the error bound of data reconstruction in Subsection III-C.

\section{A. Preliminaries And Problem Formulation}

First, we introduce some notations. Let $\mathcal{G}(\mathcal{V}, \mathcal{E}, \mathbf{W})$ denote an $\mathcal{N}$-vertex, undirected, connected, and weighted graph, where $\mathcal{V}$ is the set of vertices with $|\mathcal{V}|=\mathcal{N}, \mathcal{E}$ is the set of edges, and $\mathbf{W}$ is the weighted adjacency matrix. Graph $\mathcal{G}$ is constructed by using $K$-nearest neighbor algorithm to model the geographical adjacency of monitored points (an example of graph $\mathcal{G}$ is shown in Fig. 2). The sensed data of connected vertices on graph $\mathcal{G}$ constructed by $K$-nearest neighbor algorithm tend to have high smoothness and spatio-temporal correlation, and the quality of data reconstruction can be improved by leveraging $K$-nearest neighbor algorithm with $K=5$ [5]. Similar to [6], to suppress the influence of mal-connections and reflect the geographical adjacency quantitatively, we set the weight of connected two vertices $i$ and $j W_{i j}$ to be inversely proportional to the square of the distance $D_{i j}^{2}$, i.e., $W_{i j}=\frac{1}{D_{i j}^{2}}$; else if two vertices $i$ and $j$ are not connected, $W_{i j}=0$.

According to the theory of GSP [4], the grapgh Laplacian of $\mathcal{G}(\mathcal{V}, \mathcal{E}, \mathbf{W})$ is denoted as

$$
\mathbf{L}=\mathbf{D}-\mathbf{W}
$$

where $\mathbf{D}$ is a diagonal matrix $\operatorname{diag}\left(d_{1}, \ldots, d_{\mathcal{N}}\right)$ and $d_{i}=\sum_{j=1}^{\mathcal{N}} W_{i j}$ is the degree of vertex $i$. By using singular value decomposition, $\mathbf{L}=\mathbf{U} \boldsymbol{\Lambda} \mathbf{U}^{\mathrm{T}}$, where $\mathbf{U}$ is an eigenvector matrix $\mathbf{U}=\left[\mathbf{u}_{1}, \mathbf{u}_{2}, \ldots, \mathbf{u}_{\mathcal{N}}\right]$ and $\boldsymbol{\Lambda}$ is an eigenvalue matrix $\boldsymbol{\Lambda}=\operatorname{diag}\left(\lambda_{1}, \lambda_{2}, \ldots, \lambda_{\mathcal{N}}\right)$. And, the frequency component of a graph signal $\mathbf{x} \in \mathbb{R}^{\mathcal{N}}$ corresponding to $\lambda_{k}$ equals the inner product of $\mathbf{x}$ and $\mathbf{u}_{k}$, i.e.,

$$
\hat{x}\left(\lambda_{k}\right)=\left\langle\mathbf{x}, \mathbf{u}_{k}\right\rangle=\sum_{i=1}^{\mathcal{N}} x(i) u_{k}(i),
$$

where $\langle\cdot\rangle$ denotes taking the inner product of a matrix. Note that the smaller the eigenvalues are, the lower the frequency components of a graph signal are.

The sampling of graph signal $\mathbf{x}$ can be expressed as

$$
\mathbf{y}=\mathbf{j} \circ \mathbf{x}+\mathbf{v}
$$

where $\circ$ is the Hadamard product, $\mathbf{v}$ is the additive Gaussian white noise, and $\mathbf{j} \in\{0,1\}^{\mathcal{N}}$ is the sampling operator defined as

$$
j_{u}=\left\{\begin{array}{ll}
1, & u \in \mathcal{P} \\
0, & u \notin \mathcal{P}
\end{array},\right.
$$

where $\mathcal{P}$ is the set of all sampled vertices on graph $\mathcal{G}$. 
And, the smoothness of graph signal $\mathrm{x}$ can be evaluated by the graph Laplacian quadratic form, i.e.,

$$
\mathcal{S}_{m}(\mathbf{x})=\mathbf{x}^{\mathrm{T}} \mathbf{L} \mathbf{x}=\sum_{(i, j) \in \mathcal{E}} W_{i j}[x(j)-x(i)]^{2}=\sum_{k=1}^{\mathcal{N}} \lambda_{k} \hat{x}\left(\lambda_{k}\right)^{2} .
$$

When the value of $\mathcal{S}_{m}$ becomes smaller, the graph signal $\mathrm{x}$ is smoother.

The set of $\epsilon$-smooth graph signals on graph $\mathcal{G}$ is expressed as

$$
\Phi_{\epsilon}(\mathcal{G})=\left\{\mathbf{x}: \mathbf{x}^{\mathrm{T}} \mathbf{L} \mathbf{x} \leq \epsilon\right\}, \epsilon \geq 0,
$$

where $\epsilon$ is the smoothness level.

Then, combing (4) with (6) and introducing temporal difference signals, the data reconstruction at the $t$ th time instant can be formulated as a convex optimization problem similar to [5], i.e.,

$$
\min _{\mathbf{x}_{t}} \underbrace{\frac{1}{2}\left\|\mathbf{j}_{t} \circ \mathbf{x}_{t}-\mathbf{y}_{t}\right\|_{2}^{2}}_{\mathrm{A}}+\underbrace{\frac{\mu}{2}\left(\mathbf{x}_{t}-\hat{\mathbf{x}}_{t-1}\right)^{\mathrm{T}} \mathbf{L}\left(\mathbf{x}_{t}-\hat{\mathbf{x}}_{t-1}\right)}_{\mathrm{B}},
$$

denoted as function $f_{\mathbf{x}}\left(\mathbf{x}_{t}\right)$, where $\mu$ is the regularization parameter, $\mathbf{x}_{t}$ is the current reconstructed data, $\hat{\mathbf{x}}_{t-1}$ is the reconstructed data of the previous time, $\mathbf{j}_{t}$ is the current sampling operator, term A penalizes the error of the samples on the known vertices, and term B encourages the smoothness of temporal difference signal $\left(\mathbf{x}_{t}-\hat{\mathbf{x}}_{t-1}\right)$.

And, the gradient of function $f_{\mathbf{x}}\left(\mathbf{x}_{t}\right)$ is

$$
\nabla f_{\mathbf{x}}\left(\mathbf{x}_{t}\right)=\mathbf{j}_{t} \circ \mathbf{x}_{t}-\mathbf{y}_{t}+\mu \mathbf{L}\left(\mathbf{x}_{t}-\hat{\mathbf{x}}_{t-1}\right) .
$$

Then, letting $\nabla f_{\mathbf{x}}\left(\mathbf{x}_{t}\right)=0$, we can get the closed-form solution for (8), i.e.,

$$
\mathbf{x}_{t}^{*}=\left(\mu \mathbf{L}+\mathbf{J}_{t}\right)^{-1}\left(\mu \mathbf{L} \hat{x}_{t-1}+\mathbf{y}_{t}\right),
$$

where $\mathbf{J}_{t}=\operatorname{diag}\left(\mathbf{j}_{t}\right)$.

\section{B. CDRC Algorithm}

In (10), since the computation for the inverse matrix in many vertices on graph $\mathcal{G}$ is very hard, we try to get the closed-form solution by applying a gradient descent method.

Here, we propose a CDRC algorithm based on conjugate gradient method to solve problem (8). Next, we give more details for the CDRC algorithm, and the detailed procedure of the algorithm is shown in Algorithm 1.

In each iteration of Lines 2-8, the algorithm mainly composes two steps: 1) the determination of stepsize and 2) the update of the next search direction. Defining $\Delta \mathrm{x}_{t}^{k}$ as the search direction at the $k$ th step, the optimal stepsize $\tau$ at the $k$ th step depends on the line minimization rule: $\min _{\tau} f_{\mathbf{x}}\left(\mathbf{x}_{t}\right)\left(\mathbf{x}_{t}^{k}+\tau \Delta \mathbf{x}_{t}^{k}\right)$. By taking the partial derivative with respect to $\tau$, we have

$$
\frac{\partial f_{\mathbf{x}}\left(\mathbf{x}_{t}+\tau \Delta \mathbf{x}_{t}\right)}{\partial \tau}=\left\langle\Delta \mathbf{x}_{t}, \nabla f_{\mathbf{x}}\left(\mathbf{x}_{t}+\tau \Delta \mathbf{x}_{t}\right)\right\rangle=0
$$

Then, we can get the optimal stepsize $\tau$, i.e.,

$$
\tau=-\frac{\left\langle\Delta \mathbf{x}^{k}, \nabla f_{\mathbf{x}}\left(\mathbf{x}_{t}^{k}\right)\right\rangle}{\left\langle\Delta \mathbf{x}_{t}^{k}, \nabla f_{\mathbf{x}}\left(\Delta \mathbf{x}_{t}^{k}\right)+\mathbf{y}_{t}\right\rangle}
$$

According to the optimal stepsize $\tau$, the iterative procedure can be expressed as

$$
\mathbf{x}_{t}^{k+1}=\mathbf{x}_{t}^{k}+\tau \Delta \mathbf{x}_{t}^{k} .
$$

The search direction $\Delta \mathbf{x}_{t}^{k+1}$ is composed of 1) the gradient at the $(k+1)$ th step and 2) the search direction at the $k$ th step, i.e.,

$$
\Delta \mathbf{x}_{t}^{k+1}=-\nabla f_{\mathbf{x}}\left(\mathbf{x}_{t}^{k+1}\right)+\gamma \Delta \mathbf{x}_{t}^{k}
$$

where

$$
\gamma=\frac{\left\|\nabla f_{\mathbf{x}}\left(\mathbf{x}_{t}^{k+1}\right)\right\|_{2}^{2}}{\left\|\nabla f_{\mathbf{x}}\left(\mathbf{x}_{t}^{k}\right)\right\|_{2}^{2}}
$$


The complexity of the CDRC algorithm is $\mathcal{O}\left(\mathcal{N} \mathcal{N}_{\text {max }}\right)$, where $\mathcal{O}(\mathcal{N})$ is the calculation complexity at each iteration and $\mathcal{N}_{\max }$ is the maximum iterative time at the worst case.

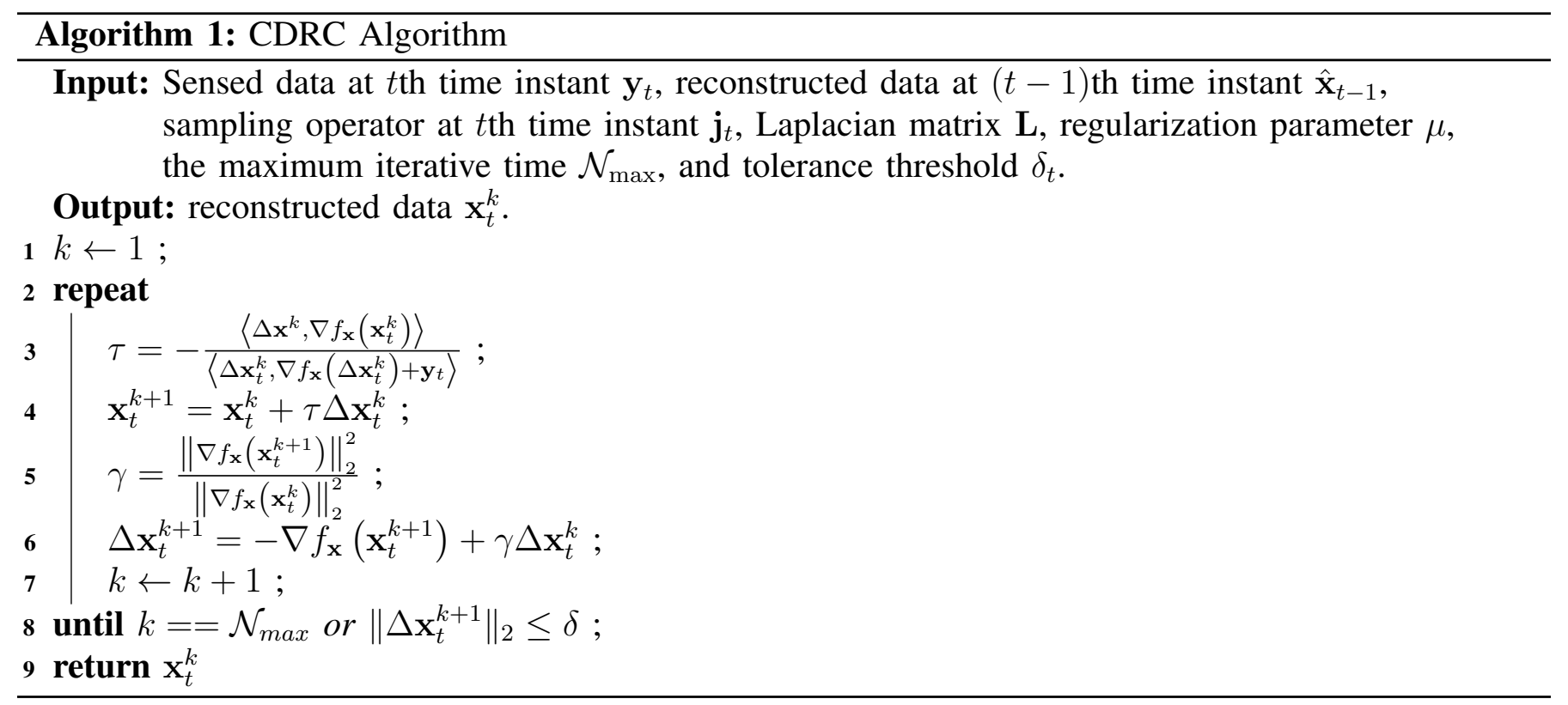

\section{Error Bound of Data Reconstruction}

In this subsection, we will show the upper bound of reconstruction error between the closed-form solution of problem (8) $\mathbf{x}_{t}^{*}$ and true data $\tilde{\mathbf{x}}_{t}$ shown in Proposition 1.

First, we define some terms: the differential signal $\tilde{\mathbf{d}}_{t}=\tilde{\mathbf{x}}_{t}-\tilde{\mathbf{x}}_{t-1}$, the reconstruction error of the $(t-1)$ th time instant $e_{t-1} \geq\left\|\mathbf{x}_{t-1}^{*}-\tilde{\mathbf{x}}_{t-1}\right\|_{2}$, the additive noise at the $t$ th time instant $n_{t} \geq\left\|\mathbf{v}_{t}\right\|_{2}$, and the bias vector $\hat{\mathbf{e}}_{t}=\mathbf{x}_{t}^{*}-\tilde{\mathbf{x}}_{t}$.

Proposition 1: if $\tilde{\mathbf{d}}_{t}$ is $\epsilon$-smooth with regard to graph $\mathcal{G}$ which has at least one vertex sampled at the $t$ th time instant, we have

and

$$
\left\|\hat{\mathbf{e}}_{t}\right\|_{2}=\left\|\mathbf{x}_{t}^{*}-\tilde{\mathbf{x}}_{t}\right\|_{2} \leq \frac{\sqrt{2\left(n_{t}^{2}+\mu \zeta_{t}^{2}\right)}+\sqrt{\mu} \zeta_{t}+n_{t}^{2}}{\lambda_{\min }}
$$

$$
\zeta_{t}=\sqrt{\epsilon}+\lambda_{\max } e_{t-1},
$$

where $\lambda_{\min }$ is the smallest singular value of matrix $\left(\mathbf{J}_{t}+\sqrt{\mu} \mathbf{L}^{1 / 2}\right)$ and $\lambda_{\max }$ is the largest singular value of matrix $\mathbf{L}^{1 / 2}$.

Proof: Here, we demonstrate Proposition 1. Based on the optimality of $\mathbf{x}_{t}^{*}$, we can get

$$
f_{\mathbf{x}}\left(\mathbf{x}_{t}^{*}\right) \leq f_{\mathbf{x}}\left(\tilde{\mathbf{x}}_{t}\right)
$$

Plugging (4) and (8) into the above inequation, we have

$$
\begin{aligned}
& \left\|\mathbf{J}_{t} \hat{\mathbf{e}}_{t}-\mathbf{v}_{t}\right\|_{2}^{2}+\mu\left(\hat{\mathbf{e}}_{t}+\tilde{\mathbf{d}}_{t}-\hat{\mathbf{e}}_{t-1}\right)^{\mathrm{T}} \mathbf{L}\left(\hat{\mathbf{e}}_{t}+\tilde{\mathbf{d}}_{t}-\hat{\mathbf{e}}_{t-1}\right) \\
& \leq\left\|\mathbf{v}_{t}\right\|_{2}^{2}+\mu\left(\tilde{\mathbf{x}}_{t}-\hat{\mathbf{x}}_{t-1}\right)^{\mathrm{T}} \mathbf{L}\left(\tilde{\mathbf{x}}_{t}-\hat{\mathbf{x}}_{t-1}\right) .
\end{aligned}
$$

Since $\left\|\mathbf{L}^{1 / 2}\left(\mathbf{x}_{t}-\hat{\mathbf{x}}_{t-1}\right)\right\|_{2}^{2}=\left(\mathbf{x}_{t}-\hat{\mathbf{x}}_{t-1}\right)^{\mathrm{T}} \mathbf{L}\left(\mathbf{x}_{t}-\hat{\mathbf{x}}_{t-1}\right)$, (19) can be rewritten as

$$
\begin{aligned}
& \left\|\mathbf{J}_{t} \hat{\mathbf{e}}_{t}-\mathbf{v}_{t}\right\|_{2}^{2}+\mu\left\|\mathbf{L}^{1 / 2}\left(\hat{\mathbf{e}}_{t}+\tilde{\mathbf{d}}_{t}-\hat{\mathbf{e}}_{t-1}\right)\right\|_{2}^{2} \\
& \leq\left\|\mathbf{v}_{t}\right\|_{2}^{2}+\mu \underbrace{\left\|\mathbf{L}^{1 / 2}\left(\tilde{\mathbf{d}}_{t}-\hat{\mathbf{e}}_{t-1}\right)\right\|_{2}^{2}}_{\text {C }} .
\end{aligned}
$$

Next, we know that the above term $\mathrm{C}$ has an upper bound, i.e.,

$$
\begin{aligned}
\left\|\mathbf{L}^{1 / 2}\left(\tilde{\mathbf{d}}_{t}-\hat{\mathbf{e}}_{t-1}\right)\right\|_{2} & \leq\left\|\mathbf{L}^{1 / 2} \tilde{\mathbf{d}}_{t}\right\|_{2}+\left\|\mathbf{L}^{1 / 2} \hat{\mathbf{e}}_{t-1}\right\|_{2} \\
& \leq \sqrt{\epsilon}+\lambda_{\max } e_{t-1}=\zeta_{t} .
\end{aligned}
$$


Furthermore, due to the property of inequation $\frac{a+b}{2} \leq \sqrt{\frac{a^{2}+b^{2}}{2}}$, (20) can be rewritten as

$$
\begin{aligned}
\left\|\mathbf{J}_{t} \hat{\mathbf{e}}_{t}-\mathbf{v}_{t}\right\|_{2}+\sqrt{\mu}\left\|\mathbf{L}^{1 / 2}\left[\hat{\mathbf{e}}_{t}-\left(\hat{\mathbf{e}}_{t-1}-\tilde{\mathbf{d}}_{t}\right)\right]\right\|_{2} \\
\leq \sqrt{2\left(n_{t}^{2}+\mu \zeta_{t}^{2}\right)} .
\end{aligned}
$$

Applying triangle inequality to (22), we have

$$
\begin{array}{r}
\left\|\mathbf{J}_{t} \hat{\mathbf{e}}_{t}\right\|_{2}-\left\|\mathbf{v}_{t}\right\|_{2}+\sqrt{\mu}\left\|\mathbf{L}^{1 / 2} \hat{\mathbf{e}}_{t}\right\|_{2}-\sqrt{\mu}\left\|\mathbf{L}^{1 / 2}\left(\hat{\mathbf{e}}_{t-1}-\tilde{\mathbf{d}}_{t}\right)\right\|_{2} \\
\leq \sqrt{2\left(n_{t}^{2}+\mu \zeta_{t}^{2}\right)} \\
\left\|\mathbf{J}_{t} \hat{\mathbf{e}}_{t}\right\|_{2}+\sqrt{\mu}\left\|\mathbf{L}^{1 / 2} \hat{\mathbf{e}}_{t}\right\|_{2} \leq \sqrt{2\left(n_{t}^{2}+\mu \zeta_{t}^{2}\right)}+\sqrt{\mu} \zeta_{t}+n_{t}^{2} \\
\left\|\left(\mathbf{J}_{t}+\sqrt{\mu} \mathbf{L}^{1 / 2}\right) \hat{\mathbf{e}}_{t}\right\|_{2} \leq \sqrt{2\left(n_{t}^{2}+\mu \zeta_{t}^{2}\right)}+\sqrt{\mu} \zeta_{t}+n_{t}^{2} .
\end{array}
$$

Then, according to (23), we have

$$
\begin{aligned}
\lambda_{\min }\left\|\hat{\mathbf{e}}_{t}\right\|_{2} & \leq\left\|\left(\mathbf{J}_{t}+\sqrt{\mu} \mathbf{L}^{1 / 2}\right) \hat{\mathbf{e}}_{t}\right\|_{2} \\
& \leq \sqrt{2\left(n_{t}^{2}+\mu \zeta_{t}^{2}\right)}+\sqrt{\mu} \zeta_{t}+n_{t}^{2} .
\end{aligned}
$$

Thus, from above inequality, we get (16), i.e.

$$
\left\|\hat{\mathbf{e}}_{t}\right\|_{2} \leq \frac{\sqrt{2\left(n_{t}^{2}+\mu \zeta_{t}^{2}\right)}+\sqrt{\mu} \zeta_{t}+n_{t}^{2}}{\lambda_{\min }} .
$$

To be noted, since the upper bound is related to parameters $\mathbf{J}_{t}$ and $e_{t-1}$, it increases with the number of unsampled points and may be accumulated over time.

\section{Simulations And Performance Evaluations}

In this section, by exploiting two real-world datasets, we conduct two simulations to explore the performance of the proposed DRC model. We set simulation parameters for the proposed CDRC algorithm as follows: 1) regularization parameter $\mu=10^{-2}$,2) the maximum number of iterations $\mathcal{N}_{\max }=10^{4}$, and 3) the tolerance threshold $\delta_{t}=10^{-6}$. Similar to [5], we take some experimental settings as follows: 1) the smoothness level $\epsilon=1,2$ ) the standard deviation of noise $n=0.1$, and 3) $K=5$ in $K$-nearest neighbor algorithm which is executed only once. The correlation range and sensing radius are respectively set to $10 \mathrm{~m}$ and $5 \mathrm{~m}$ consistent with [3]. For effectively verifying the coverage performance of the proposed model, a monitored point $p$ is covered if one of the following conditions is met: 1) $p$ within the sensing radius of one sensor (when the DC model is used) and 2) the RMSE lower than the RMSE threshold $\gamma_{t}$ (when the DRC or CIC model is used). In this letter, the coverage ratio of WSNs is calculated by

$$
\mathcal{C}_{r}=\frac{\mathcal{N}_{p c}}{\mathcal{N}_{p}}
$$

where $\mathcal{N}_{p c}$ is the number of points covered and $\mathcal{N}_{p}$ is the number of points required to be covered $\left(\mathcal{N}_{p}=\mathcal{N}\right)$, and the quality of reconstructed data at all unsampled points is evaluated by the average RMSE of reconstructed data at unsampled points:

$$
\bar{e}_{u n}=\frac{\left\|\hat{\mathbf{X}}^{u n}-\tilde{\mathbf{X}}^{u n}\right\|_{F}}{\sqrt{\mathcal{N}_{d}^{u n}}},
$$

where $\hat{\mathbf{X}}^{u n}=\left[\hat{\mathbf{x}}_{1}, \ldots, \hat{\mathbf{x}}_{\mathcal{N}_{u n}}\right]^{\mathrm{T}}$ is the matrix of the reconstructed data at unsampled points, $\tilde{\mathbf{X}}^{u n}=$ $\left[\tilde{\mathbf{x}}_{1}, \ldots, \tilde{\mathbf{x}}_{\mathcal{N}_{u n}}\right]^{\mathrm{T}}$ is the matrix of the true data at unsampled points, $\mathcal{N}_{\text {un }}$ is the number of all unsampled points, and $\mathcal{N}_{d}^{u n}$ is the length of the data at unsampled points.

\section{A. Temperature Sensor Network Dataset}

In the first simulation shown in Table I, Fig. 3, and Fig. 4, we select the temperature data of 53 sensors from dataset [7] to verify the performance of the proposed model. The dimension of the sensed data is 
TABLE I

RMSE $\bar{e}_{\text {un }}$ UNDER DIFFERENT UNSAMPLED POINTS

\begin{tabular}{|c||c|c|c|c|c|c|}
\hline Methods \Unsampled points & 10 & 15 & 20 & 25 & 30 & 35 \\
\hline Algorithm 1 & 0.662132 & 0.702915 & 0.719612 & 0.755810 & 0.795147 & 0.859001 \\
\hline The optimal solution of (10) & 0.662130 & 0.702912 & 0.719610 & 0.755809 & 0.795146 & 0.859001 \\
\hline The upper bound at the 1th time instant & 1.416187 & 1.672819 & 2.019374 & 2.444332 & 3.038857 & 4.058628 \\
\hline Average upper bound & 13.81653 & 16.33827 & 19.14484 & 23.55355 & 30.26955 & 40.75893 \\
\hline
\end{tabular}

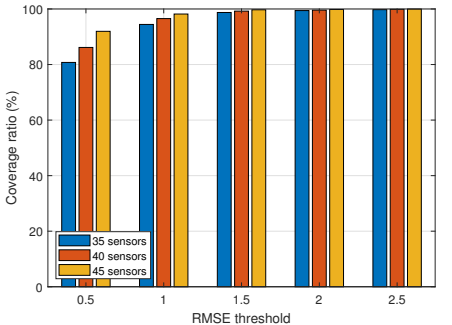

Fig. 3. Coverage ratio vs. RMSE threshold.

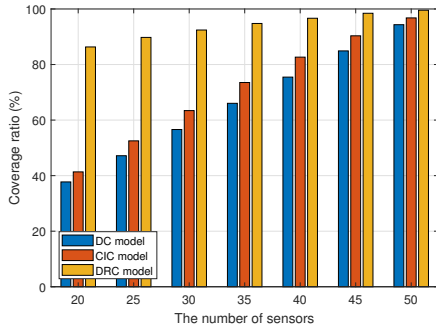

Fig. 4. Coverage ratio vs. the number of sensors.

$53 \times 500$, and the sensed data at unsampled points are missing due to sensor malfunctions. The range of temperature values is from $14.01{ }^{\circ} \mathrm{C}$ to $28.0{ }^{\circ} \mathrm{C}$, and the interval of time instants is 30 seconds.

As shown in Table I, we illustrate the RMSE of reconstructed data at all unsampled points $\bar{e}_{\text {un }}$ under different methods via changing the number of unsampled points. We can see that Algorithm 1 and the optimal solution of (10) have the similar performance, and the RMSE increases with the number of unsampled points.

Fig. 3 shows the coverage ratio under different sensor quantities (35, 40, and 45 sensors) by varying the RMSE threshold when the proposed model is used. Observably, the coverage ratio under the DRC model increases with the number of sensors or the RMSE threshold. And, the gap of the coverage ratio between three quantities of sensors narrows as the RMSE threshold increases.

As shown in Fig. 4, we depict the coverage ratio under different methods via changing the number of sensors. Here, we set RMSE threshold $\gamma_{t}$ to 1.0. The coverage ratio of the proposed DRC model is higher than that of the DC model and CIC model. With the increment of the number of sensors, the coverage radio under all models increases, and the coverage radio under the proposed model is close to $100 \%$ gradually.

\section{B. Global Sea-Level Pressure Dataset}

In the second simulation shown in Table II, Fig. 5, and Fig. 6, we select the global sea-level pressure data from dataset [8] to verify the performance of the proposed DRC model. We randomly select the data of 100 points over 600 sampling instants from the dataset. The dimension of the data is $100 \times 600$. The range of pressure values is from $96.22 \mathrm{kPa}$ to $110.06 \mathrm{kPa}$, and the interval of time instants is five days.

As shown in Table II, we show the RMSE of reconstructed data at all unsampled points $\bar{e}_{u n}$ under different methods by varying the number of unsampled points. We can observe that the performances of

TABLE II

RMSE $\bar{e}_{\text {un }}$ UNDER DIFFERENT UNSAMPLED POINTS

\begin{tabular}{|c||c|c|c|c|c|c|}
\hline Methods \Unsampled points & 10 & 20 & 30 & 40 & 50 & 60 \\
\hline Algorithm 1 & 0.746687 & 0.806494 & 0.845760 & 0.895832 & 0.957504 & 1.019326 \\
\hline The optimal solution of (10) & 0.746478 & 0.806401 & 0.845686 & 0.895828 & 0.957483 & 1.019264 \\
\hline The upper bound at the 1th time instant & 7.788567 & 8.427240 & 8.935114 & 9.119517 & 9.399783 & 9.464195 \\
\hline Average upper bound & 55.15752 & 62.17484 & 68.32077 & 72.05092 & 76.30751 & 80.48206 \\
\hline
\end{tabular}




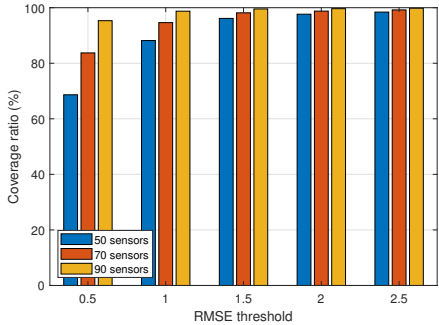

Fig. 5. Coverage ratio vs. RMSE threshold.

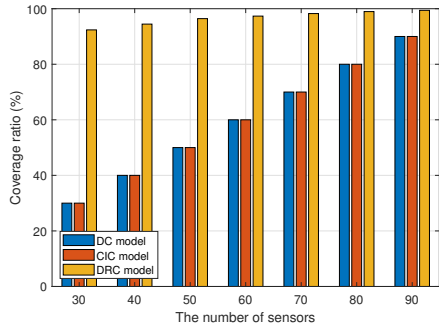

Fig. 6. Coverage ratio vs. the number of sensors.

Algorithm 1 and the optimal solution of (10) are very close, and the RMSE increases as the number of unsampled points.

Fig. 5 depicts the coverage ratio on different sensor quantities (50, 70, and 90 sensors) by changing the RMSE threshold when the proposed model is used. We can see that the coverage ratio increases with the number of sensors or the RMSE threshold. And, the gap of the coverage ratio between three quantities of sensors narrows as the RMSE threshold increases.

As shown in Fig. 6, we illustrate the coverage ratio under different models via changing the number of sensors. Here, we set RMSE threshold $\gamma_{t}$ to 1.5 because the pressure values are greater than the temperature values in Subsection IV-A. Compared with the DC model and CIC model, the coverage ratio with the proposed model has better performance. The performance of the CIC model is the same as the DC model since the distance between unsampled points and sensors is far beyond the correlation range. As the number of sensors increases, the coverage radio under all models increases, and the coverage radio under our proposed model is close to full coverage gradually.

\section{CONCLUSION}

In this letter, we mainly propose an efficient coverage model to improve sensing coverage of WSNs. Motivated from the theory of GSP, we develop a novel notion, DRC. Unlike the existing DC model and CIC model, our proposed model does not rely on the fixed sensing radius/correlation range of sensors. Based on the theory of GSP, the model can jointly reconstruct missing data at unsampled points by exploiting the proposed CDRC algorithm which fully utilizing the smoothness of temporal difference signals as well as the graph Laplacian matrix, without adding any sensors. Also, by applying real-world datasets, numerical simulations demonstrate that the proposed model can further improve the coverage ratio of the WSN and reduces the required number of sensors, compared with the DC model and CIC model.

\section{REFERENCES}

[1] Z. Fei, B. Li, S. Yang, et al., "A survey of multi-objective optimization in wireless sensor networks: metrics, algorithms, and open problems," IEEE Commun. Surveys Tuts., vol. 19, no. 1, pp. 550-586, First Quarter 2017.

[2] X. Bai, Z. Yun, D. Xuan, et al., "Optimal multiple-coverage of sensor networks," in Proc. IEEE Int. Conf. Comput. Commun. (INFOCOM), Shanghai, China, pp. 2498-2506, Apr. 2011.

[3] X. Deng, Z. Tang, L. T. Yang, et al., "Confident information coverage hole healing in hybrid industrial wireless sensor networks," IEEE Trans. Industr. Inform., vol. 14, no. 5, pp. 2220-2229, May 2018.

[4] D. I. Shuman, S. K. Narang, P. Frossard, et al., "The emerging field of signal processing on graphs: extending high-dimensional data analysis to networks and other irregular domains," IEEE Signal Process. Mag., vol. 30, no. 3, pp. 83-98, May 2013.

[5] K. Qiu, X. Mao, X. Shen, et al., "Time-varying graph signal reconstruction,” IEEE J. Sel. Top. Signal Process., vol. 11, no. 6, pp. 870-883, Sept. 2017.

[6] X. Mao, K. Qiu, T. Li, et al., "Spatio-temporal signal recovery based on low rank and differential smoothness," IEEE Trans. Signal Process., vol. 66, no. 23, pp. 6281-6296, 1 Dec. 2018.

[7] Sensor Network Data, Mar. 30, 2016. [Online]. Available: http://www.cs.cmu.edu/guestrin/Research/Data/

[8] Sea-Level Pressure, 1948-2010, May 25, 2016. [Online]. Available: http://research.jisao.washington.edu/data_sets/reanalysis/ 